\title{
P02.63. Efficacy of an 8-week online mindfulness stress management program in a corporate call center
}

\author{
D Allexandre ${ }^{1 *}$, A Neuman $^{1}$, J Hunter ${ }^{1}$, T Morledge ${ }^{2}$, M Roizen $^{1}$ \\ From International Research Congress on Integrative Medicine and Health 2012 \\ Portland, Oregon, USA. 15-18 May 2012
}

\section{Purpose}

Stress is inherent in our society and results in great suffering, increased healthcare cost, and impaired work performance. An online program can offer a cost effective and practical solution that can help cope better with stress. In this study, we evaluated the effectiveness of an 8-week online mindfulness stress reduction (OSR) program in reducing work related stress and burnout and in improving well-being in a corporate call center. We also evaluated whether 1-hour weekly group practice and experience sharing at the workplace would improve program retention and engagement.

\section{Methods}

161 participants were randomized either to wait-list control (CTL, N=37), OSR ( $=54)$ or OSR and weekly group meetings (OSR+grp, $\mathrm{N}=70)$. The Perceived Stress Scale, Maslach Burnout Inventory (professional efficacy and exhaustion subscales), Mindful Attention Awareness Scale and SF36 (emotional well-being and role functioning subscales) were administered at baseline, post intervention and at 8-week follow-up.

\section{Results}

We observed overall a greater post intervention decrease in stress and exhaustion and increase in mindfulness, emotional well-being and role functioning in the OSR+grp (cohen $\mathrm{d}=1.3,0.8,0.6,1.4$ respectively) compared to OSR $(\mathrm{d}=1,0.4,0.5,0.8)$ and wait-list control $(\mathrm{d}<0.4)$. This overall improvement was also maintained at follow-up for most measures. The improvement was significantly greater for the OSR compared to CTL for stress and emotional well-being, and for OSR+grp compared to CTL for all outcomes except for professional efficacy. OSR+grp improved significantly more than OSR for stress, emotional wellbeing and emotional role functioning. Weekly group practice significantly increased program engagement and reduced dropout rate (13\% for OSR+grp compared to $55 \%$ for OSR).

\section{Conclusion}

An online mindfulness stress management program when combined with weekly group practice can offer a practical and cost-effective approach to decrease stress and burnout and improve mindfulness and well-being at the workplace.

\section{Author details \\ ${ }^{1}$ Cleveland Clinic, Cleveland, USA. ${ }^{2}$ Revati Wellness, Cleveland, USA.}

Published: 12 June 2012

doi:10.1186/1472-6882-12-S1-P119

Cite this article as: Allexandre et al.: P02.63. Efficacy of an 8-week online mindfulness stress management program in a corporate call center. BMC Complementary and Alternative Medicine 2012 12(Suppl 1):P119.

Cleveland Clinic, Cleveland, USA

Full list of author information is available at the end of the article 